\title{
PREVISÃO ESTATÍSTICA E COMPARAÇÃO DE DADOS DA MORTALIDADE POR COVID-19 NO BRASIL E ESTADOS UNIDOS
}

\author{
Camille Schmidt de Proença ${ }^{a}$ \\ Carla Adriana Pizarro Schmidt b \\ a Centro Universitário Fundação Assis Gurgacz - FAG, Avenida das Torres, 500, Cascavel-PR, Brasil, \\ csproenca@minha.fag.edu.br \\ b DAPRO - Departamento de Administração e Produção, Universidade Tecnológica Federal do Paraná, Av. Brasil, \\ 4232, Medianeira-PR, Brasil, carlaschmidt@utfpr.edu.br
}

\section{RESUMO}

A utilidade da modelagem é permitir a construção diária de uma previsão confiável para a evolução das doenças, bem como, acompanhar o desenvolvimento dos fenômenos; armazenando o estudo histórico das ocorrências, em relação às ações de contenção utilizadas naquele dado momento. Uma pandemia ocasionada por um tipo específico de coronavírus abalou o mundo em 2020-2021, sendo que o presente estudo se propôs a realizar uma análise estatística comparativa de dados do número de mortes confirmadas quinzenalmente, ocasionadas pela COVID-19, em valores corrigidos por milhão de habitantes, coletados entre outubro de 2020 e maio de 2021, construir e apresentar modelos capazes de se ajustar às séries temporais de valores e oferecer uma previsão estatística do cenário para alguns dias no Brasil e Estados Unidos da América (EUA). Os modelos foram desenvolvidos sendo que para o Brasil o melhor ajuste foi de um modelo ARIMA $(2,2,3)$ e para os EUA um modelo de suavização exponencial de Holt Winters aditivo. A tendência foi de queda nos valores, sendo que no Brasil a redução prevista em média iria de 145 a 109 e para os EUA de 27 para 25, ao longo dos 21 dias analisados, mostrando que a previsão apresentada para a situação da COVID-19 no Brasil, no momento da realização deste estudo foi mais grave, podendo no decorrer do tempo, mesmo com uma população menor que a americana acumular mais mortes, o que é preocupante para o histórico de enfrentamento dessa enfermidade em nosso país.

Palavras-chave: Coronavírus; COVID-19; Pandemia; Séries Temporais.

\footnotetext{
*Autor correspondente: Carla Adriana Pizarro Schmidt, doutora em Agronomia pela Universidade Estadual de Londrina e professora titular 40h DE da Universidade Tecnológica Federal do Paraná junto ao Departamento de Administração e Produção. Endereço: Av. Brasil, 4232, Medianeira - Paraná, Brasil, (45) 3240-8000 ramal 8135. carlaschmidt@utfpr.edu.br
} 


\section{INTRODUÇÃO}

Um tipo de coronavírus, detectado em 31 de dezembro de 2019 na China, mais especificamente na cidade de Wuhan, denominado como SARS-CoV-2, resultou em uma pandemia, pois a doença causada pelo vírus; a COVID-19, se espalhou rapidamente por todas as nações do mundo e resultou em milhões de mortes (LANA et al., 2020; BAENINGER et al., 2020). Especialmente países como o Brasil, Estados Unidos da América (EUA), Índia e Rússia, vêm enfrentando uma gravidade maior na situação, com elevada quantidade tanto de acometidos quanto de pessoas mortas (PAPASTEFANOPOULOS et al., 2020).

A aplicação de análises estatísticas para a previsão futura de séries temporais de acordo com Booth e Tickle (2008), podem ser utilizadas na previsão de mortalidade, mesmo esses modelos tendo sido destinados a previsões de curto prazo eles têm se mostrado úteis na realização dessas antevisões mesmo a longo prazo. Benvenuto et al. (2020) construíram modelos ARIMA, que são modelos autorregressivos integrados a médias móveis, para a previsão da propagação da doença, com base em dados diários de prevalência e incidência da COVID-19, fornecidos pela Universidade Johns Hopkins.

Talkhi et al. (2021) avaliaram nove possíveis técnicas de modelagem, sendo que dentre elas também testaram a qualidade das previsões apresentadas pelos modelos ARIMA e de suavização exponencial. Realizaram a previsão do número de casos confirmados e de óbitos que ocorreriam por conta da evolução da doença no Irã. O modelo de suavização exponencial de Holt-Winter se mostrou como o mais adequado para a previsão de casos de mortes futuras, enquanto o método de rede MLP foi o mais indicado para previsão dos casos confirmados.

De acordo com Setel et al. (2020), a vigilância em tempo real dos dados da magnitude da pandemia ocasionada pela
COVID-19, bem como o acompanhamento das taxas de infecção e mortalidade, é muito importante, com vistas a estabelecer e dimensionar ações necessárias a serem tomadas pelos formuladores de políticas públicas.

A principal utilidade da modelagem, seria possibilitar às instituições envolvidas na saúde pública a construção diária de uma previsão confiável para a epidemia da COVID-19, além de permitir o acompanhamento e avaliação dos principais planos estratégicos de controle da infecção, tais como distanciamento $e$ quarentena, avaliando se estes estariam realmente sendo eficientes (BENVENUTO et al., 2020; PAPASTEFANOPOULOS et al., 2020; TALKHI et al., 2021; LYNCH; GORE, 2021).

Ter ciência da situação com antecedência traz uma enorme vantagem, pois elucida a necessidade ou não do fechamento e melhor planejamento para adoção de medidas restritivas, bem como para o conhecimento e preparo para possíveis surtos futuros em outros locais, dessa forma fica clara a utilidade das técnicas de previsão de cenários futuros da doença (SETEL et al., 2020; TALKHI et al., 2021).

Diante de tal contexto este estudo se propõe a realizar uma análise estatística comparativa da mortalidade no Brasil e EUA, países mais gravemente afetados até $O$ momento, no período de ocorrência da segunda onda da doença, entre os meses de outubro de 2020 e maio de 2021 e apresentar modelos capazes de se ajustarem às séries temporais de dados, apresentando com base nestes o cenário a ser esperado com fundamento na previsão estatística das taxas de mortalidade para alguns dias nos dois países.

\section{MATERIAL E MÉTODOS}

O presente estudo utilizou-se apenas de dados documentais secundários, os quais já são de domínio público, não sendo possível a 
identificação dos sujeitos. Por esse motivo, a aprovação do Comitê de Ética em Pesquisa não foi necessária. Os dados foram coletados diretamente do gráfico da série histórica temporal de novas mortes, confirmadas quinzenalmente, ocasionadas pela COVID-19, em valores corrigidos por milhão de habitantes, disponibilizado pelo site Our Word in Data (2021), entre os meses de outubro de 2020 até abril de 2021, época em que a segunda onda de contaminação atingiu esses dois países, totalizando 212 dias de acompanhamento de dados. As mortes referem-se ao número cumulativo de mortes confirmadas nas duas semanas (quinzena), anteriores à data em que o valor é apresentado.

Optou-se por acompanhar os dados de mortalidade, pois, tanto no Brasil quanto nos EUA, de acordo com Martins et al. (2020) e Papastefanopoulos et al. (2020), a quantidade de testes realizados para confirmação dos casos da doença tem sido muito pequena para se saber 0 exato número de pessoas acometidas; ficando, portanto, esse valor subnotificado, o que dificulta a correta estimativa e previsão do cenário real, baseando-se apenas no número de pessoas comprovadamente doentes.

Depois de digitados em planilha os dados foram submetidos a uma análise estatística descritiva, com auxílio do software Action Stat ${ }^{\circledR}$ (Portal Action, 2020), para o melhor entendimento da série temporal. Esse mesmo programa foi ainda utilizado na avaliação das séries em relação a presença de tendência, sazonalidade e estacionareidade.

Um gráfico BoxPlot foi construído no software livre GretL (BAIOCCHI; DISTASO, 2003), o qual foi também utilizado para auxílio nas análises de séries temporais, principalmente na realização das análises pelos modelos ARIMA. Modelos ARIMA incluem a parte do modelo autorregressiva (AR), com uma integração (I) a parte do modelo de média móvel (MA). Para construção desses modelos deve-se estimar 3 parâmetros $(p, d, q)$, os quais nesse estudo foram obtidos com base nos gráficos da função de autocorrelação (ACF) e nos correlogramas de autocorrelação parcial (PACF).
O software NNQ (Núcleo de Normalização e Qualimetria, 2020), desenvolvido e fornecido gratuitamente pelo Núcleo de Normalização e Qualimetria da UFSC, o qual disponibiliza as análises por meio de todos os modelos de suavização exponencial disponíveis, desde a simples até a tripla, apresentados por Hyndman e Athanasopoulos (2020), foram testados.

Após a realização da previsão pelos modelos de suavização exponencial e pelos modelos ARIMA, os resultados dos erros MAPE foram comparados e os modelos com os menores erros foram escolhidos para validação. Para tanto foi construída uma previsão para 10 dias e validada contra os dados reais, na sequência os dois melhores modelos foram escolhidos e uma previsão completa para os demais 21 dias do mês de maio foi apresentada, para os dois países acompanhados.

\section{RESULTADOS E DISCUSSÃO}

Papastefanopoulos et al. (2020) em seu estudo de previsão acompanharam a situação dos EUA, Brasil, Índia e Rússia, os autores alertaram para a possibilidade de ocorrência de um pico pandêmico em outubro de 2020 e destacam a tendência de piores cenários para esses países, por conta das elevadas taxas de transmissão observadas naquele estudo. Os seis países mais populosos do mundo são: China, Índia, EUA, Indonésia, Paquistão e Brasil, nessa exata ordem (SEPULVEDA; ROBLES, 2021). Eles foram comparados na Figura 1 e pôde-se observar que as taxas de mortalidade ocasionadas pela Covid-19 foram muito diferentes nesses seis países.

Enquanto países como China, Paquistão e Indonésia não mostraram muita elevação em suas taxas de mortalidade, o Brasil e EUA apresentaram taxas muito elevadas, ficando ainda a Índia em uma situação intermediária, que vem piorando desde o início do mês de maio de 2021. Com base na Figura 1 pode-se notar que tanto EUA quanto Brasil foram muito mais duramente afetados pela pandemia do que os outros países com grandes populações, quando se leva em conta o número de mortes 
diárias ajustadas quinzenalmente por milhão de pessoas. Atualmente o Brasil está muito à frente nesse quesito, tendo inclusive ultrapassado os EUA. A Índia vem desde o início de maio de 2021 mostrando um crescimento preocupante na mortalidade $\mathrm{e}$ pode-se ainda observar que a segunda onda da doença foi claramente mais mortal nesses 3 países.

Papastefanopoulos et al. (2020) explicam que: deve-se entender que está além da

Figura 1. Série histórica temporal de novas mortes ocasionadas pela COVID-19 por milhão de habitantes nos seis Países mais populosos do Mundo confirmadas quinzenalmente desde fevereiro de 2020 quando iniciou a pandemia.

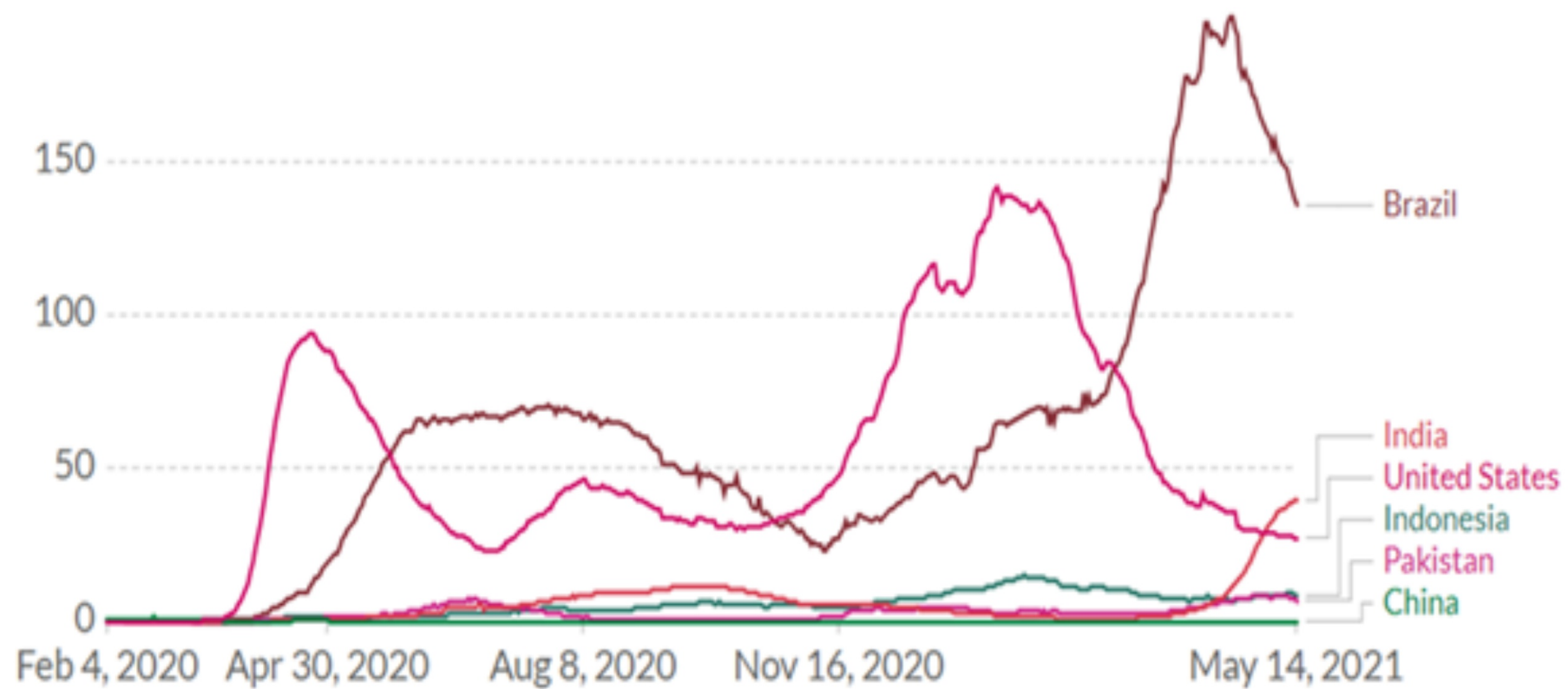

Fonte: OUR WORD IN DATA, site que apresenta dados coletados do Repositório de dados da COVID-19 do Centro de Ciência e Engenharia de Sistemas (CSSE) da Universidade de Johns Hopkins, (2021).

capacidade de qualquer país testar toda sua população. Mas, eles destacam que a imposição de bloqueio parcial nas cidades, reduzir e evitar viagens internacionais, fechar shoppings, teatros e academias, poderia ajudar muito no controle da pandemia. Souza et al. (2021), ao analisarem a dinâmica espacial da dispersão da COVID-19 no Brasil, especialmente no estado da Bahia, concluíram que os aeroportos e rodovias que cruzam o estado contribuíram para a interiorização da doença, o que reforça a necessidade de controlar a movimentação das pessoas para conter a disseminação da doença.

Papastefanopoulos et al. (2020), destacam ainda que medidas tais como manter obrigatório o uso de máscara em público e a proibição de grandes reuniões, bem como incentivar as pessoas a ficarem em casa, sempre que possível, também deve ser recomendado. Alertam ainda que os centros de saúde e hospitais devem gerenciar o fluxo de pacientes de perto, observando bem a possibilidade de superlotação e disponibilidade de leitos e de acordo com eles as universidades e outras instituições educacionais devem ser encorajadas a continuar sempre que possível com os métodos de e-learning, onde o ensino é realizado a distância por meio das ferramentas digitais.

O menor valor de mortes por milhão no período acompanhado entre os meses de outubro de 2020 e abril de 2021, totalizando 7 meses de acompanhamento de dados diários, foi de 22,84, ocorrido no Brasil no dia 10 novembro de 2020, enquanto o menor valor dos EUA foi de 29,04, que ocorreu no dia 30 de abril de 2021. Os valores mais elevados e alarmantes de mortalidade encontrados aconteceram no Brasil, sendo que inclusive pode-se observar pontos discrepantes 
ilustrados na Figura 2, o maior valor encontrado no período foi de 197,26 ocorrido no dia 19 de abril de 2021, enquanto o maior valor encontrado para os EUA foi de 141,82 encontrado no dia 17 de janeiro de 2021.

As médias de mortalidade para os dois países são aproximadas e foram respectivamente de 73,59 para os EUA e 76,99 para o Brasil, que em média vem apresentando uma pior situação. A assimetria dos dois conjuntos de dados foi positiva, indicando que a média representada na Figura 2 por um ponto no centro da caixa, foi maior que a mediana, indicada pela linha que atravessa a caixa do BoxPlot. A mediana encontrada para as taxas de mortalidade dos EUA foi levemente superior, devido ao fato dos dados estarem mais compactados, com uma faixa menor entre o maior e o menor valor, o que também resultou em um valor menor de amplitude $(112,78)$ quando comparado ao resultado obtido para o Brasil $(174,42)$.

O coeficiente de variação encontrado para os dados Brasileiros foi 0,69 superior ao encontrado para os EUA de 0,51, o que já era esperado devido a presença de outliers na série de dados de mortalidade encontrados no Brasil. Cabe destacar que a variação pode ser considerada muito elevada e isso ocorre devido a rapidez de evolução no quadro da doença com crescimento notadamente exponencial de ocorrência, acompanhado com quadros onde a doença se agrava e o paciente morre ampliando a mortalidade no mesmo sentido.

T. BoxPlot representativo das mortes confirmadas quinzenalmente por milhão de habitantes, ocasionadas pela COVID-19 entre os meses de outubro de 2020 e abril 2021 no Brasil (BR) e Estados Unidos da América (EUA).

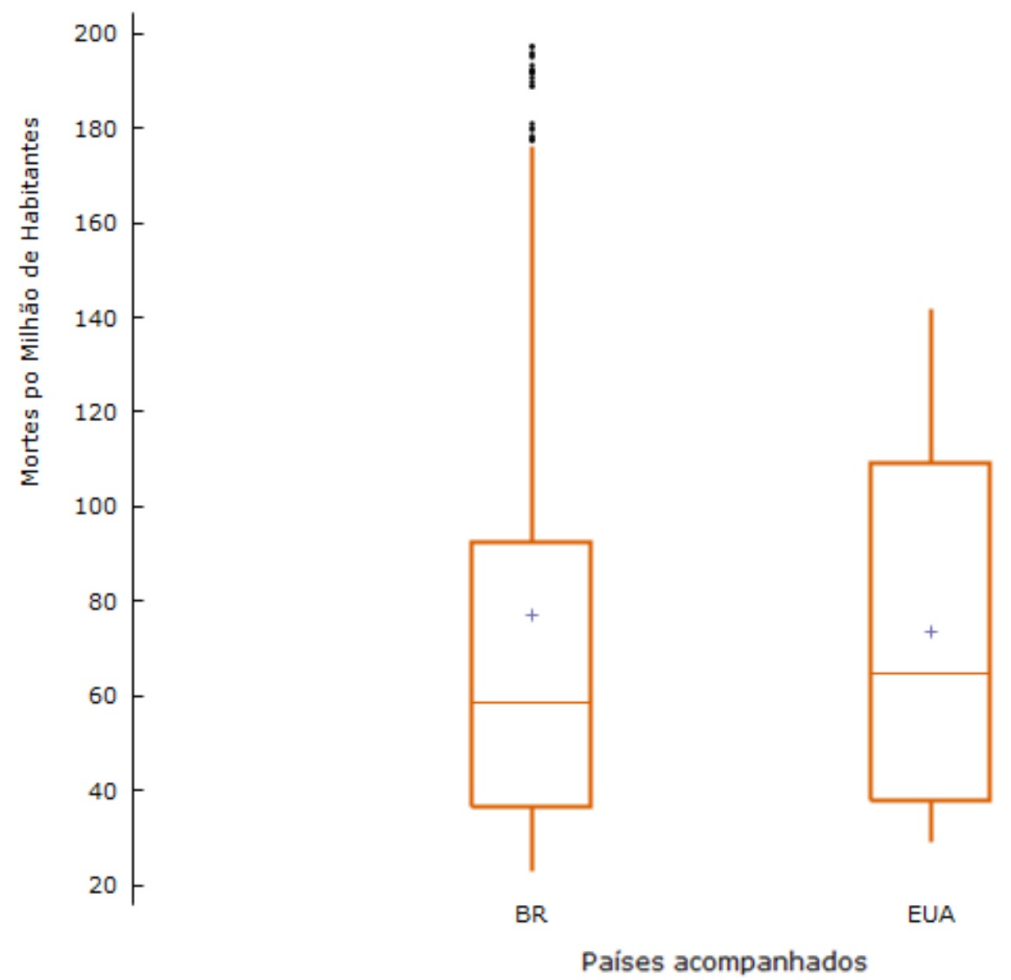

Fonte: Autoria Própria com base em dados disponibilizados pela Universidade Johns Hopkins

As séries temporais de dados ao longo do período acompanhado podem ser visualizadas na Figura 3, nota-se que o Brasil nos últimos meses março e abril de 2021, viveu a sua pior situação e já ultrapassou 180 mortes confirmadas quinzenalmente por milhão de pessoas. Devido ao tamanho da população brasileira essa quantidade de pessoas que morreram em decorrência da Covid-19 é bastante significativa e pode chegar a ser a maior quantidade em relação a todos os outros países, pois apesar de vários países da Europa terem chegado a taxas de mortalidade até superiores, eles são menos populosos e os 
outros países, tão populosos quanto o Brasil, não chegaram a taxas tão elevadas, nem mesmo os EUA que parou em valores inferiores, quando a curva epidêmica recuou (Figura 3).

De acordo com Scafetta (2020), os padrões de correlação encontrados sugerem que a letalidade da COVID-19 piora significativamente (4 vezes em média) sob temperaturas climáticas entre $4{ }^{\circ} \mathrm{C}$ e $12{ }^{\circ} \mathrm{C}$ juntamente com as condições meteorológicas de baixa umidade relativa, entre $59 \%$ e $73 \%$. O autor explica que esse clima pode tanto favorecer a propagação do vírus da COVID-19, quanto agravar a suscetibilidade das pessoas à uma pneumonia secundária, o que no final ocasiona os resultados encontrados no estudo, de um número superior de mortes naquela situação climática. Tanto climas mais frios quanto mais quentes, bem como umidades relativas diferentes das citadas, têm sido responsáveis pela redução nas mortes.

Figura 3. Série temporal representativa do número de mortes por mil habitantes confirmadas quinzenalmente pela COVID-19 entre os meses de outubro de 2020 e abril 2021 no Brasil (BR) e Estados Unidos da América (EUA).

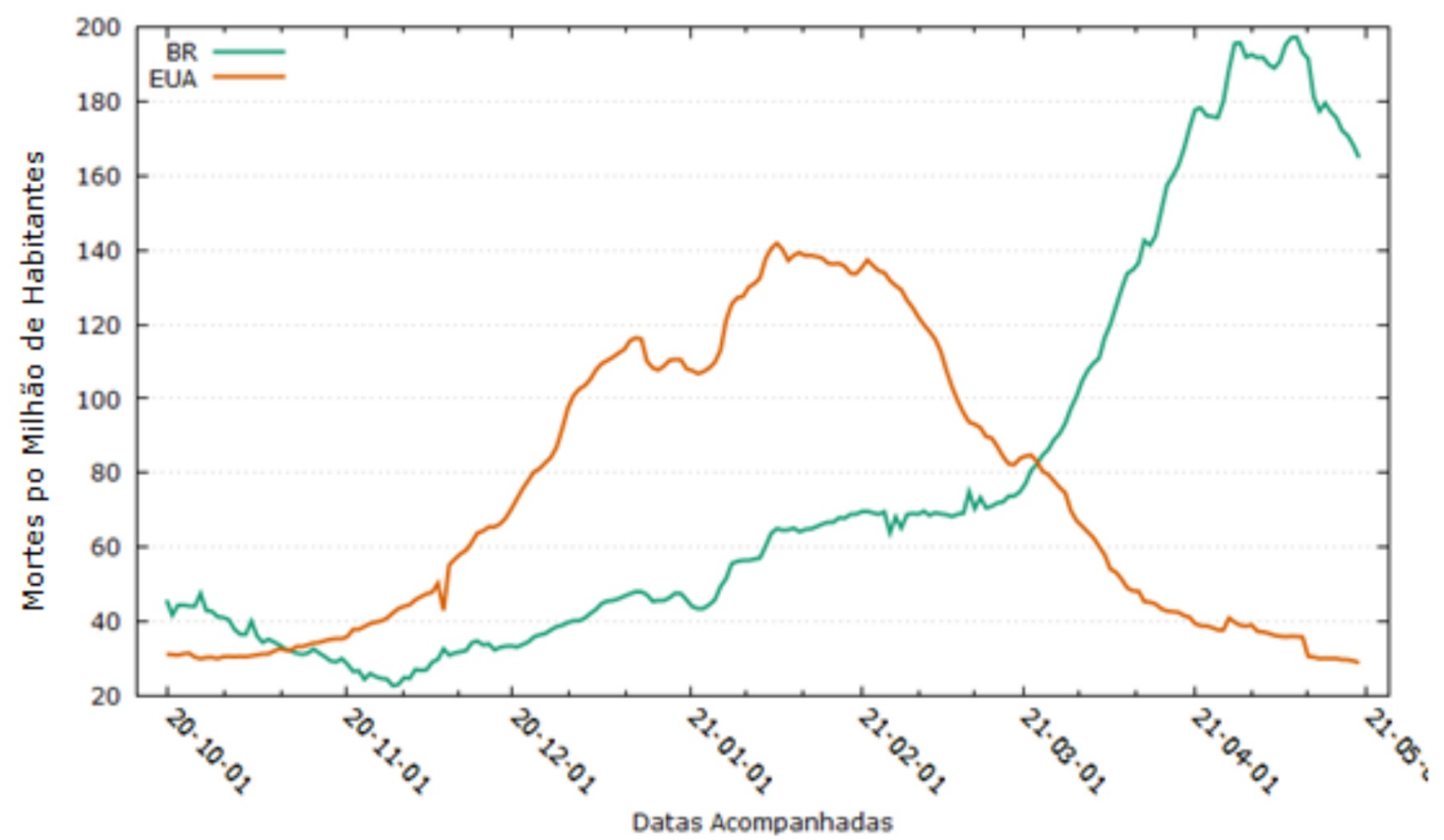

Fonte: Autoria Própria com base em dados disponibilizados pela Universidade Johns Hopkins

Tal fato pode explicar as épocas de pico distintas sendo que nos EUA o pico da segunda onda ocorreu entre os meses de dezembro e março, época de temperaturas mais frias, enquanto no Brasil a piora ocorreu a partir de abril. Então, apesar de nesse momento a curva estar com uma tendência de redução dos casos de morte, deve-se ainda manter o estado de alerta em relação a doença no Brasil, pois o período de inverno em grande parte do país ainda se estenderá por mais alguns meses e a previsão de estiagem também tem sido noticiada para grande parte do país.

As duas séries temporais mostraram tanto tendência, quanto sazonalidade a qual foi confirmada pelos testes de Kruskal-Wallis e
Friedman e os dados foram considerados não estacionários com base nos testes de KPPS, Dickey-Fuller Aumentado e Phillips-Perron. Os resultados sazonais devem-se a contabilização ao longo dos dias da semana, isso pode ser mais relacionado a dinâmica de informação das mortes do que a morte real da pessoa ter ocorrido naquele exato dia da semana, mas para prever o que realmente será informado nos próximos dias é uma avaliação interessante e pode ajudar na aproximação da previsão dos dados reais.

Papastefanopoulos et al. (2020) explicam que existem padrões sazonais nos dados, mas que esses são artificiais e não confirmam o número real de infecções daquele dia ou um efeito de maiores e menores infecções em 
determinados dias da semana. Isso pode acontecer por causa das flutuações na alimentação dos bancos de dados por conta da disponibilização das informações. Aqueles autores notaram que nos EUA, ocorria uma baixa notificação de doentes na quarta-feira e uma alta no sábado enquanto no Brasil, ocorria elevação do número de doentes na sexta-feira e sábado e redução na segunda-feira. No presente estudo observou-se índices sazonais superiores de mortes para os dias de sábado e domingo no Brasil e segunda-feira para os EUA enquanto os inferiores foram observados na terça-feira para o Brasil e sexta-feira para os EUA.

O melhor modelo de suavização exponencial que apresentou os menores erros de previsão para o Brasil foi o que tratou $\circ$ nível, a sazonalidade e a tendência todas de forma aditiva com valores de alfa 0,99 ; de beta 0,16 e gama 0,01 ; enquanto o melhor modelo ARIMA foi o ARIMA $(2,2,3)$. Ao se avaliar os resultados previstos pelo modelo de suavização e pelo modelo ARIMA $(2,2,3)$ para - Brasil observou-se que O modelo ARIMA $(2,2,3)$ apresentou os menores erros e uma previsão muito mais próxima que os modelos de suavização exponencial, como se pode observar nas Figuras 4 e 5 que ilustram os resultados das previsões realizadas por esses dois modelos.

Figura 4. Resultados dos valores mínimos e máximos e previstos pelo modelo de suavização exponencial aditivo para os 10 primeiros dias do mês de maio, comparados com os valores reais observados para o Brasil.

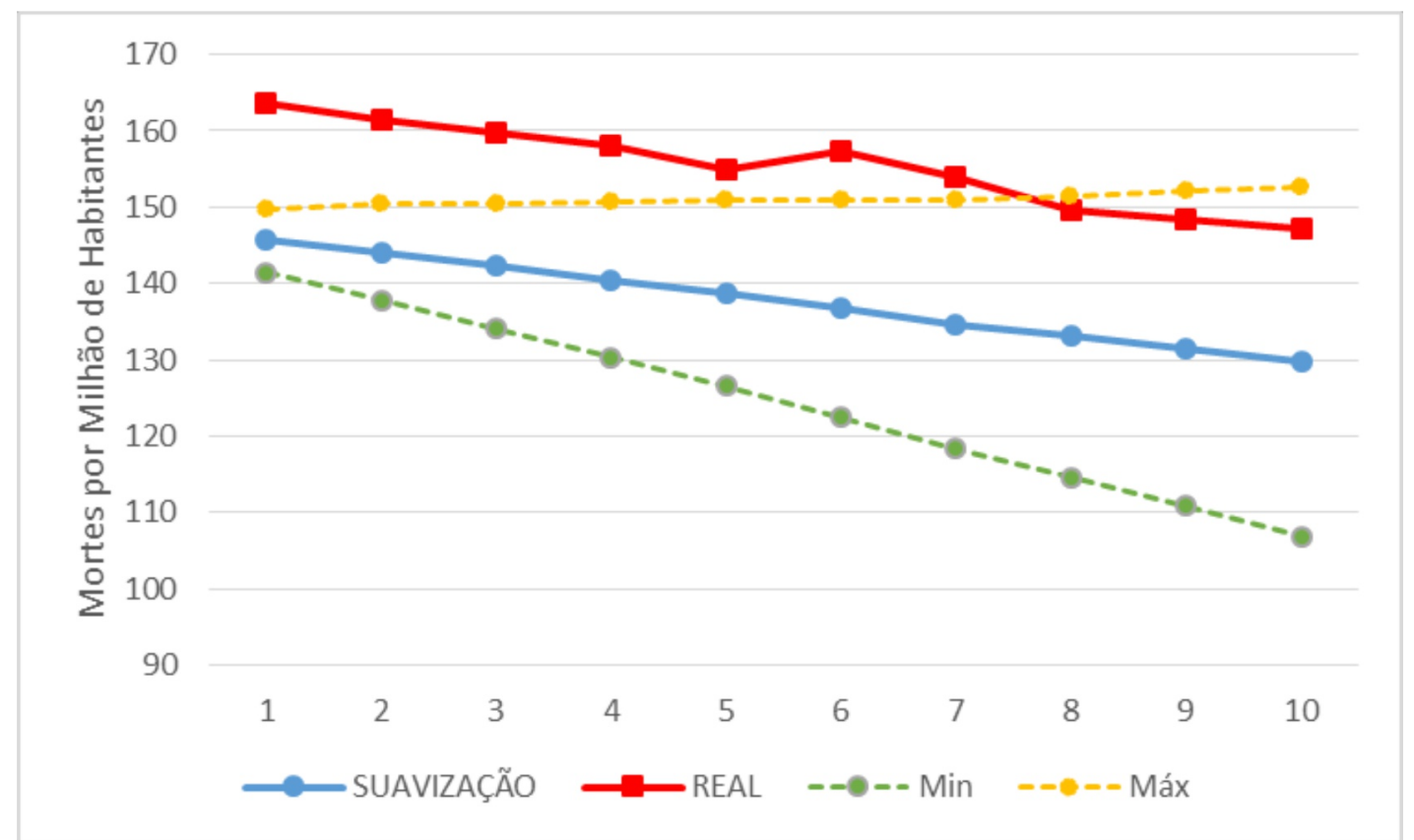

Fonte: Autoria Própria.

Percebeu-se que os valores previstos pelo modelo de suavização exponencial foram muito inferiores aos valores reais observados e que os dados reais nem mesmo estiveram dentro dos limites da previsão (Figura 4). Enquanto os valores previstos pelo modelo ARIMA $(2,2,3)$ ficaram muito próximos para os primeiros 5 dias e levemente inferiores para os demais, mas todos os valores reais ficaram dentro dos limites da previsão.

De acordo com Papastefanopoulos et al.
(2020), os modelos ARIMA e SEIR são os mais conhecidos e amplamente usados na previsão de séries temporais de epidemias, então não é surpresa os bons resultados observados na previsão do modelo ARIMA $(2,2,3)$. Benvenuto et al. (2020) também apresentaram modelos ARIMA um pouco diferentes $(1,0,4)$ e $(1,0,3)$ porém igualmente bem-sucedidos, para a previsão da propagação da COVID-19 com base em dados diários de prevalência e incidência da doença. O que confirma a 
possibilidade de bons ajustes e boa previsão estimativa de dados epidêmicos. com base nesses tipos de modelos econométricos, quando são aplicados para a

Figura 5. Resultados dos valores mínimos e máximos e previstos pelo modelo ARIMA-2-2-3 para os 10 primeiros dias do mês de maio, comparados com os valores reais observados para o Brasil.

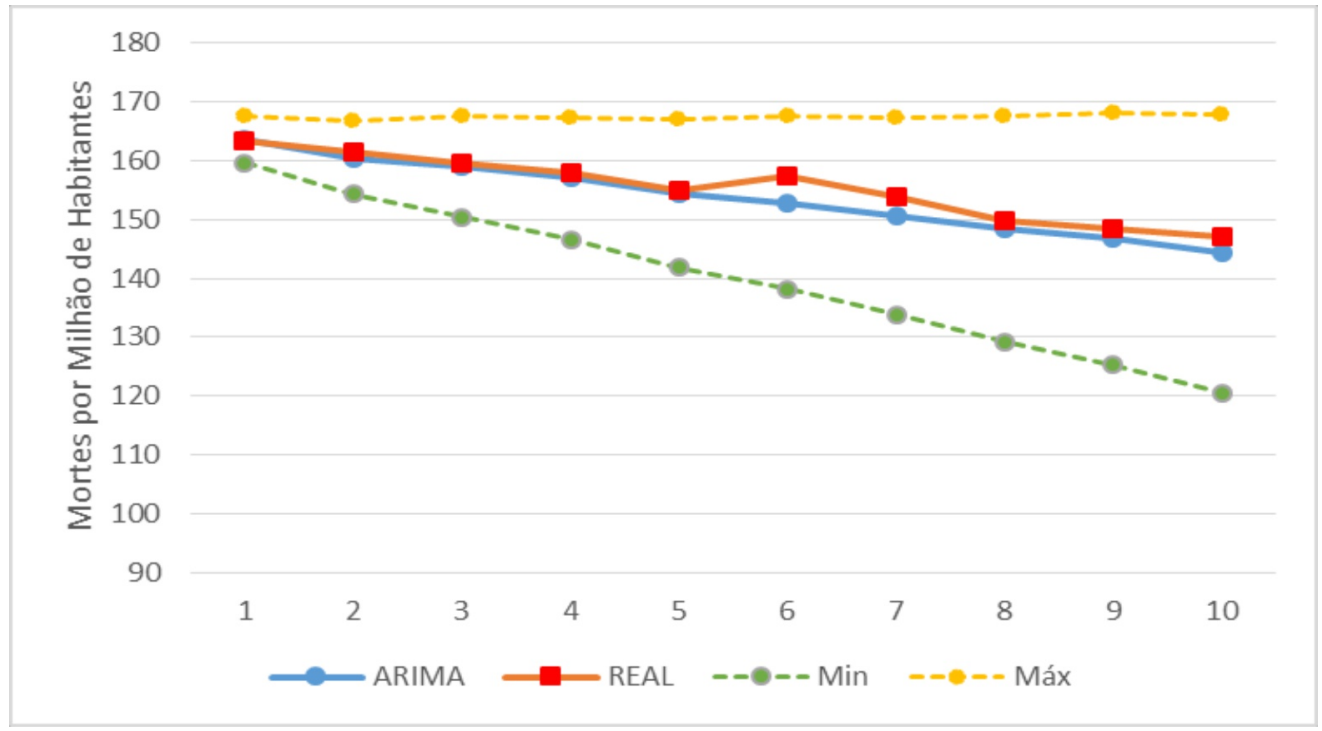

Fonte: Autoria Própria.

O melhor modelo de suavização dados reais, encontram-se ilustrados nas exponencial que apresentou os menores erros Figuras 6 e 7 . Percebeu-se que; para os de previsão para os EUA foi o que tratou o dados dos EUA, tanto o modelo de suavização nível, a sazonalidade e a tendência todas de exponencial, quando o $\operatorname{ARIMA}(0,2,1)$, foram forma aditiva, porém com o amortecimento da validados, apresentando uma boa proximidade tendência, com valores de alfa 0,99; beta 0,22, dos dados reais, os quais ainda estiveram gama 0,01 ; e fi 0,95 ; enquanto o melhor dentro dos limites inferior e superior de modelo ARIMA foi o ARIMA $(0,2,1)$. Os previsão, fornecidos pelos modelos escolhidos. resultados para previsão comparadas aos

Figura 6. Resultados dos valores mínimos e máximos e previstos pelo modelo de suavização exponencial aditivo para os 10 primeiros dias do mês de maio, comparados com os valores reais observados para os EUA.

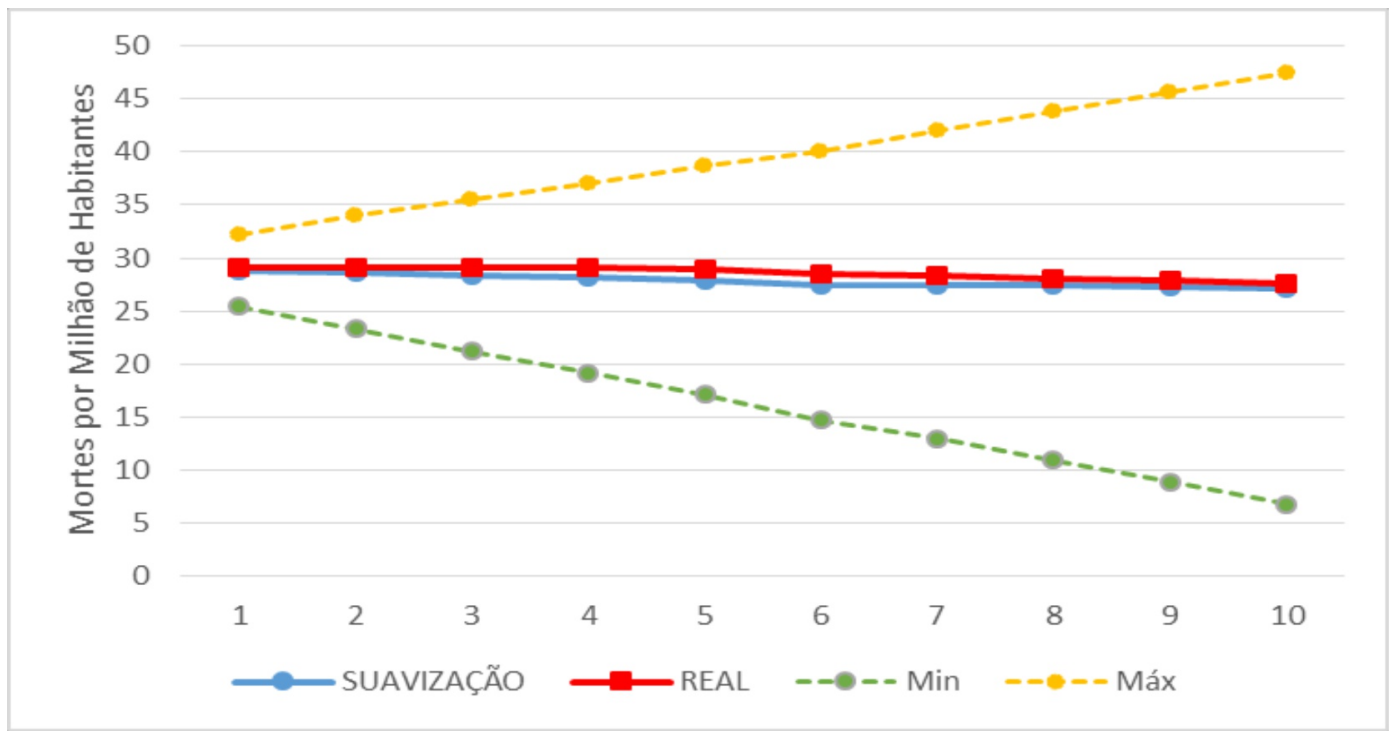

Fonte: Autoria Própria. 
Talkhi et al. (2021), também verificaram um bom ajuste no modelo de suavização exponencial (Holt-Winters) para previsão futura de ocorrência de mortes pela COVID-19 no Irã, sendo que naquele momento do estudo daqueles autores os valores da série temporal também se apresentavam praticamente estáveis, com leve queda, muito semelhante a situação que está ocorrendo nos EUA no período avaliado no presente estudo. Isso leva a crer que nessas situações os modelos de suavização exponencial tenham uma tendência de acertar mais e apresentar menores erros de previsão.

Figura 7. Resultados dos valores mínimos e máximos e previstos pelo modelo ARIMA-0-2-1 para os 10 primeiros dias do mês de maio, comparados com os valores reais observados para os EUA.

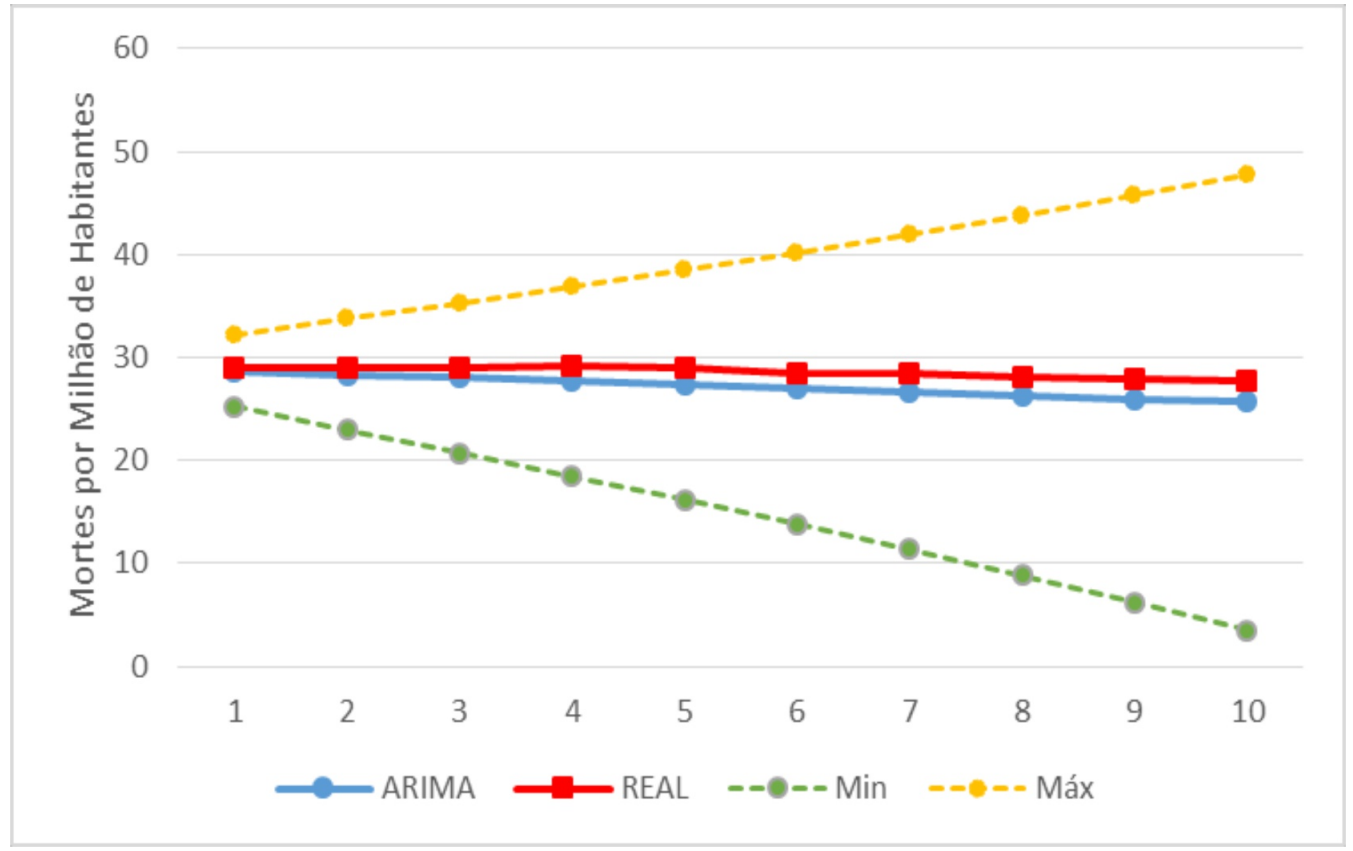

Fonte: Autoria Própria.

Logo após essa validação os resultados reais para os 10 primeiros dias do mês de maio foram incluídos na série e foi realizada nova previsão para os últimos 21 dias do mês de maio pelos melhores modelos, sendo que para o Brasil optou-se pelo uso do modelo ARIMA $(2,2,3)$ e para os EUA optou-se pelo uso do modelo de suavização exponencial de HoltWinters AAdA, que trata nível, tendência e sazonalidade de forma aditiva e realiza um amortecimento da tendência. Os resultados obtidos para previsão para os próximos 21 dias, os valores dos coeficientes dos modelos de previsão e os resultados dos limites superiores e inferiores de previsão foram apresentados nos Quadros 1 a 4.

Os erros calculados para o modelo ARIMA $(2,2,3)$ utilizado para previsão dos valores de mortes por mil habitantes confirmadas quinzenalmente pela COVID-19 no Brasil foram: erro percentual médio de 2,14; erro médio 1,36 e erro quadrático médio de 1,977, sendo que o $U$ de Theil foi de 0,82 inferior a 1,0 o que confirma a boa qualidade desse modelo de previsão e a possibilidade de sua aplicação na previsão das mortes para os próximos dias.

Cabe destacar que devido a elevada tendência de baixa observada qualquer alteração na tendência dessa curva não seria identificada pelo modelo e precisaria ser identificada nos dados reais, por isso uma previsão estatística não deve ser realizada para horizontes de tempo muito logos, porém esta quando acompanhada pela realimentação com dados reais e feita para poucos dias pode ser muito útil no acompanhamento do andamento da evolução da pandemia e apoiar a tomada de decisão por parte dos órgãos governamentais que gerenciam as ações ligadas a saúde pública.

No atual momento meados do mês de 
maio de 2021 o Brasil contabiliza desde o início da pandemia aproximadamente $440 \mathrm{mil}$ pessoas mortas, sendo que vacinou completamente apenas $9,4 \%$ de sua população e aproximadamente $20 \%$ da população recebeu apenas a primeira dose, como a vacina foi liberada apenas para profissionais da saúde e pessoas de mais idade, em geral acima de 65 anos, pode-se dizer que ainda existem muitas pessoas vulneráveis à doença (MAPA, 2021).

Porém, acredita-se que devido a vacinação dos idosos acima de 65 anos estar bem adiantada, a redução nas mortes pode estar sendo ocasionada por esse motivo, pois pessoas idosas são mais sujeitas a mortes ocasionadas pela doença do que a população mais jovem. Tal observação se deve ao fato que a quantidade de pessoas infectadas pelo vírus da COVID-19, o SARS-Cov-2, no Brasil não está se reduzindo na mesma velocidade da redução observada nas mortes, pelo contrário, de acordo com dados da mesma fonte utilizada na previsão desse estudo a quantidade de pessoas confirmadas com a doença no presente mês (maio 2021) está ainda se elevando.

Tal suposição pode ser confirmada pelo estudo de Haas et al. (2021), onde observaram que a morte decorrente da COVID-19 em Israel caiu de $6,6 \%$ em pessoas não vacinadas para $0,2 \%$ em pessoas completamente vacinadas ( 2 doses), quando se acompanhou a população acima de 65 anos de idade naquele país.

Os valores dos coeficientes autorregressivos (AR 1 e 2 ) e de médias móveis (MA 1, 2 e 3) encontram-se disponibilizados no Quadro 1, destaca-se que se utilizou 2 integrações no modelo que foi o ARIMA $(2,2,3)$. As mortes médias confirmadas quinzenalmente referem-se ao número cumulativo de mortes confirmadas nas duas semanas anteriores, as previsões realizadas para o Brasil ainda são de valores elevados, na faixa de 145 a 109, que ainda podem ocorrer no decorrer do final do mês de maio de 2021.

Quadro 1. Coeficientes do modelo ARIMA $(2,2,3)$ para previsão dos valores de mortes por mil habitantes confirmadas quinzenalmente pela COVID-19 no Brasil.

\begin{tabular}{|c|c|}
\hline Coeficientes & Valores \\
\hline ar1 & $-1,219289063$ \\
\hline ar2 & $-0,807146894$ \\
\hline ma1 & 0,44258332 \\
\hline ma2 & $-0,089153538$ \\
\hline ma3 & $-0,845534624$ \\
\hline
\end{tabular}

Tabela 3 - Mediana das variáveis pré e pós COMAFA, Curitiba/PR, Brasil, 2020

O presente estudo foi realizado no mês de maio de 2021 e os valores reais disponibilizados no site Our Word In Data até o presente momento encontram-se dentro dos limites inferiores e superiores da previsão apresentados para o Brasil no Quadro 2. Os erros calculados para o modelo de Suavização Exponencial AAdA utilizado para previsão das mortes por mil habitantes confirmadas quinzenalmente pela COVID-19 no EUA foram: erro percentual médio de 1,64; erro médio 1,04 e erro quadrático médio de 1,72 , sendo que o $U$ de Theil foi de 0,84 inferior a 1,0 o que confirma a boa qualidade desse modelo de previsão e a possibilidade de sua aplicação na previsão das mortes para os próximos dias. 
Quadro 2. Previsões para os próximos 21 dias pelo modelo ARIMA $(2,2,3)$ para previsão dos valores de mortes por mil habitantes confirmadas quinzenalmente pela COVID-19 no Brasil

\begin{tabular}{|c|c|c|c|}
\hline Data & Previsão & Limite Inf. 95\% & Limite Sup. 95\% \\
\hline $11 / \mathrm{mai}$ & 145,8906 & 141,9819 & 149,7993 \\
\hline $12 / \mathrm{mai}$ & 143,5237 & 137,3644 & 149,683 \\
\hline $13 / \mathrm{mai}$ & 141,984 & 133,5092 & 150,4588 \\
\hline 14/mai & 140,2731 & 129,994 & 150,5522 \\
\hline $15 /$ mai & 138,1033 & 125,6993 & 150,5072 \\
\hline 16/mai & 136,6312 & 122,11 & 151,1525 \\
\hline $17 / \mathrm{mai}$ & 134,6788 & 118,1584 & 151,1992 \\
\hline 18/mai & 132,7489 & 113,9528 & 151,5449 \\
\hline 19/mai & 131,1792 & 110,1852 & 152,1733 \\
\hline 20/mai & 129,1521 & 105,907 & 152,3973 \\
\hline $21 / \mathrm{mai}$ & 127,392 & 101,7272 & 153,0569 \\
\hline $22 / \mathrm{mai}$ & 125,6756 & 97,65237 & 153,6989 \\
\hline $23 / \mathrm{mai}$ & 123,6904 & 93,18719 & 154,1937 \\
\hline 24/mai & 121,9977 & 88,9357 & 155,0597 \\
\hline 25/mai & 120,1653 & 84,5553 & 155,7753 \\
\hline 26/mai & 118,2671 & 79,98323 & 156,5511 \\
\hline 27/mai & 116,5619 & 75,57425 & 157,5496 \\
\hline 28/mai & 114,6745 & 70,94649 & 158,4026 \\
\hline 29/mai & 112,8535 & 66,28503 & 159,422 \\
\hline 30/mai & 111,0986 & 61,67293 & 160,5243 \\
\hline 31/mai & 109,2095 & 56,86217 & 161,5569 \\
\hline
\end{tabular}

Tabela 3 - Mediana das variáveis pré e pós COMAFA, Curitiba/PR, Brasil, 2020

Os parâmetros da equação de tendência (fi) e sazonalidade (gama) suavização exponencial AAdA para o nível encontram-se apresentados no Quadro 3. (alfa), tendência (beta), amortecimento de

Quadro 3. Parâmetros do modelo de Suavização Exponencial AAdA para previsão dos valores de mortes por mil habitantes confirmadas quinzenalmente pela COVID-19 nos EUA

\begin{tabular}{|c|c|}
\hline Parâmetros & Valores \\
\hline alfa & 0,99 \\
\hline beta & 0,27 \\
\hline gama & 0,01 \\
\hline fi & 0,93 \\
\hline
\end{tabular}

Fonte: Autoria Própria.

No momento avaliado a pandemia nos EUA está em um patamar de estabilidade não mostrando muita tendência e apesar dos valores estarem em leve queda a previsão (Quadro 4) demonstra que os valores devem se manter entre 24 e 27 mortes por milhão de habitantes quinzenalmente avaliadas.

No total os EUA já contabilizam aproximadamente 580 mil mortes ocasionadas pela COVID-19. Nos EUA o número de pessoas que vêm apresentando a doença 
também está caindo, então a tendência daquele país deve ser para uma futura redução também no número de mortes em decorrência da doença. Cabe destacar que de acordo com Duarte (2021), metade dos adultos já recebeu a primeira dose do imunizante e cerca de $30 \%$ da população americana já está totalmente vacinada, bem como adolescentes acima de 12 anos também estão sendo vacinados. $O$ software NNQ não conseguiu calcular os limites com 95\% de confiança sendo que o maior valor de confiança conseguido para os limites superior e inferior das previsões foi de $80 \%$.

Quadro 4. Previsões para os próximos 21 dias pelo modelo AAdA de suavização exponencial para previsão dos valores de mortes por mil habitantes confirmadas quinzenalmente pela COVID-19 nos EUA.

\begin{tabular}{|c|c|c|c|}
\hline Data & Previsão & $\begin{array}{c}\text { Limite Inf. } \\
80 \%\end{array}$ & Limite Sup. 80\% \\
\hline 11/mai & 27,19016 & 24,98046 & 29,39985 \\
\hline $12 / \mathrm{mai}$ & 26,72858 & 23,20558 & 30,25158 \\
\hline 13/mai & 26,14527 & 21,34618 & 30,94437 \\
\hline 14/mai & 26,41753 & 20,33598 & 32,49908 \\
\hline $15 / \mathrm{mai}$ & 26,54469 & 19,16697 & 33,9224 \\
\hline $16 /$ mai & 26,59227 & 17,90576 & 35,27878 \\
\hline $17 / \mathrm{mai}$ & 26,40383 & 16,39927 & 36,40839 \\
\hline $18 /$ mai & 26,03656 & 14,69814 & 37,37498 \\
\hline 19/mai & 25,66067 & 12,99805 & 38,32328 \\
\hline 20/mai & 25,15669 & 11,17112 & 39,14227 \\
\hline 21/mai & 25,50238 & 10,19795 & 40,80681 \\
\hline $22 / \mathrm{mai}$ & 25,69752 & 9,08076 & 42,31428 \\
\hline 23/mai & 25,80803 & 7,887514 & 43,72855 \\
\hline 24/mai & 25,67785 & 6,463834 & 44,89187 \\
\hline $25 / \mathrm{mai}$ & 25,36451 & 4,860946 & 45,86807 \\
\hline $26 / \mathrm{mai}$ & 25,03854 & 3,266372 & 46,81071 \\
\hline 27/mai & 24,58078 & 1,553645 & 47,60791 \\
\hline 28/mai & 24,96924 & 0,701517 & 49,23697 \\
\hline 29/mai & 25,20399 & $-0,28941$ & 50,69738 \\
\hline 30/mai & 25,35116 & $-1,35256$ & 52,05488 \\
\hline $31 / \mathrm{mai}$ & 25,25492 & $-2,64348$ & 53,15331 \\
\hline
\end{tabular}

Fonte: Autoria Própria.

Em resumo pode-se perceber que a pandemia no Brasil está em um nível pior que os EUA nesse momento, o qual já vive uma estabilização no número de mortes, e tem apresentado um valor bem menor na quantidade de mortes por mil habitantes confirmadas quinzenalmente que as apresentadas pelo Brasil.

Desta forma, apesar dos EUA contabilizarem no momento um valor total de mortes pela COVID-19 superior, ele pode acabar sendo superado pelos valores de mortos no Brasil. Cabe destacar que a população brasileira é menor que a americana e que se levarmos em conta o número de mortos corrigido pelo número de habitantes o Brasil já supera os valores de mortos americanos, porém outros países ainda contabilizam quantidades superiores de mortes proporcionais ao total de população, mas devido ao fato de possuírem populações menores o Brasil teve uma quantidade superior de pessoas mortas pela doença. 


\section{CONCLUSÃO}

Os modelos avaliados foram capazes de realizar uma previsão para alguns dias do andamento da pandemia nos dois países, tanto - Brasil quando o EUA. Novas previsões podem ser realizadas de tempos em tempos com vistas a alimentar os modelos desenvolvidos com novos dados reais, obtendo-se novas previsões para os dois países.

A aplicação dos modelos na previsão e apresentação dos dados de mortes esperadas, pode auxiliar tanto no entendimento do cenário atual da doença, quanto para alertar os órgãos governamentais, caso a situação comece a mudar de direção e volte a subir, momento no qual teriam que ser tomadas medidas mais restritivas.

\section{CONFLITO DE INTERESSE:}

Não existe nenhum tipo de conflito de interesse

\section{REFERÊNCIAS}

BAENINGER , R.; VEDONATO, R.; NANDY, S. (Coord.) Migrações internacionais e a pandemia de Covid-19. Campinas : Núcleo de Estudos de População "Elza Berquó »- Nepo/Unicamp, 2020. 636p. ISBN : 978-65-87447-06-3 (versão digital) Disponível em : https://repositorio.usp.br/directbitstream/ 04b3ca3e-828a-4f43-bd0f-cb4b3fdaa279/ miginternacional.pdf\#page=95. Acesso em : 25 out. 2021.

BAIOCCHI, G., DISTASO, W. GRETL: software econométrico para a geração GNU. J. Appl. Econom. , v.18, pp. 105 - 110, 2003.

BENVENUTO, D.; GIOVANETTI, M.; VASSALLO, L.; ANGELETTI, S.; CICCOZZI, M. Application of the ARIMA model on the COVID-2019 epidemic dataset. Data In Brief, [S.L.], v. 29, p. 105340, abr. 2020. Elsevier BV. http://dx.doi.org/10.1016/j.dib.2020.105340
DUARTE, L. EUA : Vacinação contra covid-19 é ampliada a todos os adultos a partir de hoje. UOL de 17 abr. 2021 Disponível em : https://noticias.uol.com.br/ultimas-noticias/ rfi/2021/04/19/vacinacao-contra-covid-19-eampliada-a-todos-os-adultos-nos-eua.htm? cmpid=copiaecola. Acesso em: 10 mai. 2021.

HAAS, E. J. ; ANGULO, F. J.; MCLAUGHLIN, J. M.; ANIS, E.; SINGER, S. R.; KHAN, F.; BROOKS, N.; SMAJA, M.; MIRCUS, G.; PAN, K. Impact and effectiveness of mRNA BNT162b2 vaccine against SARSCoV-2 infections and COVID-19 cases, hospitalisations, and deaths following a nationwide vaccination campaign in Israel : an observational study using national surveillance data. The Lancet, [S.L.], v. 397, n. 10287, p. 1819-1829, maio 2021. Elsevier BV. http:// dx.doi.org/10.1016/s0140-6736(21)00947-8.

LANA, R. M.; COELHO, F. C.; GOMES, M. F. da C.; CRUZ, O. G.; BASTOS, L. S.; VILLELA, D. A. M.; CODEÇO, C. T. Emergência do novo coronavírus (SARS-CoV2) e o papel de uma vigilância nacional em saúde oportuna e efetiva. Cadernos de Saúde Pública, [S.L.], v. 36, n. 3, p. 1-5, 2020. FapUNIFESP (SciELO). http://dx.doi.org/ 10.1590/0102-311x00019620.

LYNCH, C. J. ; GORE, R. Application of one-, three-, and seven-day forecasts during early onset on the COVID-19 epidemic dataset using moving average, autoregressive, autoregressive moving average, autoregressive integrated moving average, and naïve forecasting methods. Data In Brief, [S.L.], v. 35, p. 106759, abr. 2021. Elsevier BV. http:// dx.doi.org/10.1016/j.dib.2021.106759

MAPA da vacinação contra Covid-19 no Brasil Acompanhe a evolução da imunização. G1 de 19 de mai. 2021. Disponível em : https://especiais.g1.globo.com/ bemestar/vacina/2021/mapa-brasil-vacinacovid/. Acesso em : 20 mai. 2021.

MARTINS, V. L. M.; LERMEN, F. H.; MAGALHÃES, R. F. de; MATIAS, G. de S. 
Artificial Neural Networks to access curve behavior of COVID-19 in Brazil: a learning experience based on other countries. Produto \& Produção, [S.L.], v. 21, n. 3, p. 76-90, 30 set. 2020. Universidade Federal do Rio Grande do Sul. http://dx.doi.org/10.22456/19838026.103261.

NNQ - Núcleo de Normalização e Qualimetria. Previsão. Universidade Federal de Santa Catarina. Disponível em: https:// qualimetria.ufsc.br/publicacoes/software/ previsao/. Acesso em: 10 mar. 2021.

OUR WORD IN DATA. Biweekly confirmed COVID-19 cases per million people. Disponível em : https:// ourworldindata.org/grapher/biweekly-coviddeaths-per-million-people?

tab=chart\&country $=$ USA $\sim$ BRA $\sim C H N \sim$ IND IDN PAK. Acesso em : 12 mai. 2021.

PAPASTEFANOPOULOS, V.; LINARDATOS, P.; KOTSIANTIS, S. COVID-19: a comparison of time series methods to forecast percentage of active cases per population. Applied Sciences, [S.L.], v. 10, n. 11, p. 3880, 3 jun. 2020. MDPI AG. http:// dx.doi.org/10.3390/app10113880.

PORTAL ACTION. Action Stat. Disponível em: http://www.portalaction.com.br/. Acesso em 20 abr. 2021.

SCAFETTA, N. Distribution of the SARSCoV-2 Pandemic and Its Monthly Forecast Based on Seasonal Climate Patterns. International Journal Of Environmental Research And Public Health, [S.L.], v. 17, n. 10, p. 3493, 17 maio 2020. MDPI AG. http:// dx.doi.org/10.3390/ijerph17103493.

\section{SEPULVEDA, A.; ROBLES T. Ranking} demográfico: os países e cidades mais populosos do mundo. 15 de fev. 2021. Disponível em: <https://www.tempo.com/ noticias/actualidade/paises-e-cidades-com-

mais-habitantes-no-mundo-aumentodemografico-da-populacao.html>. Acesso em: 11 mai. 2021.
SETEL, P.; ABOUZAHR, C.; ATUHEIRE, E. B.; BRATSCHI, M.; CERCONE, E.; CHINGANYA, O.; CLAPHAM, B.; CLARK, S. J.; CONGDON, C.; SAVIGNY, D. Mortality surveillance during the COVID-19 pandemic. Bulletin Of The World Health Organization, [S.L.], v. 98, n. 6, p. 374-374, 1 jun. 2020. WHO Press. http://dx.doi.org/10.2471/blt.20.263194.

SOUZA, C. D. F.; MACHADO, M. F.; SILVA JUNIOR, A. G.; NUNES, B. E. B. R.; CARMO, R. F. do. Airports, highways and COVID-19: an analysis of spatial dynamics in brazil. Journal Of Transport \& Health, [S.L.], v. 21, p. 101067, jun. 2021. Elsevier BV. http:// dx.doi.org/10.1016/j.jth.2021.101067.

TALKHI, N.; FATEMI, N. A.; ATAEI, Z.; NOOGHABI, M. J. Modeling and forecasting number of confirmed and death caused COVID-19 in IRAN: a comparison of time series forecasting methods. Biomedical Signal Processing And Control, [S.L.], v. 66, p. 102494, abr. 2021. Elsevier BV. http:// dx.doi.org/10.1016/j.bspc.2021.102494. 